\title{
Analytical Assessment of DC Components Generated by Renewable Energy Resources with Inverter-Based Interconnection System due to Even Harmonics
}

\author{
Marios N. Moschakis, ${ }^{1}$ Vasilis V. Dafopoulos, ${ }^{1}$ \\ Emmanuel S. Karapidakis, ${ }^{2}$ and Antonis G. Tsikalakis ${ }^{2}$ \\ ${ }^{1}$ Department of Electrical Engineering, Technological Educational Institute of Larissa, 41110 Larrisa, Greece \\ ${ }^{2}$ Department of Natural Resources \& Environment, Technological Educational Institute of Crete, \\ Romanou 3 Street, 73133 Chania, Crete, Greece
}

Correspondence should be addressed to Marios N. Moschakis, marios.moschakis@gmail.com

Received 29 April 2012; Accepted 7 August 2012

Academic Editors: E. R. Bandala, S. Dai, and G. Namkoong

Copyright (c) 2012 Marios N. Moschakis et al. This is an open access article distributed under the Creative Commons Attribution License, which permits unrestricted use, distribution, and reproduction in any medium, provided the original work is properly cited.

\begin{abstract}
This paper deals with the assessment of DC components generated by renewable energy resources with inverter-based interconnection system to the electric grid. DC injection is a critical issue related to power quality of distribution network systems with high penetration of inverter-based interconnection systems. This type of interface systems may improve the performance of the electric generation unit and affect positively or negatively the power quality of the distribution network depending on the proper or improper designation. The investigation of the various causes of DC components and the analytical assessment of their maximum levels are crucial for the proper operation of inverter-based interface systems and the limitation of DC injection. A method based on analytical calculations using a computer software has been implemented for the assessment of DC components contained on an inverter's output voltage when even harmonics are present on the network voltage. Moreover, a simulation package was used to demonstrate the existence of DC components under various conditions. It was proved by the current analysis that the amounts of DC components generated when even harmonics are present on the network voltage can be high under abnormalities on the power grid but they are not considerable under normal operating conditions.
\end{abstract}

\section{Introduction}

The need for analysis and assessment of DC components on voltage and/or current, which may appear at electric grids, aroused mainly due to the significant problems they result in. Power transformers are the power system element which is mainly affected by the presence of DC components. Saturation, generation of harmonics, power losses, reduction in life cycle, and increasing noise levels are some of the effects on transformers introduced by the presence of DC components. Moreover, the saturation of current transformers used by measurement devices of control and protection of electric grid equipment may cause inaccurate measurements with considerable consequences.

In cases where many DC injection sources are connected to the grid, the need for limitation and standardization becomes urgent. Such cases include the interconnection of several distributed generation units at Medium-Voltage (MV) or Low-Voltage (LV) level through inverters based on the technology of power electronics. Those inverters may generate DC components on the output voltage during abnormal conditions that will lead to DC current injection flowing to the electric grid if no power transformer is used between the inverter and the grid (transformer-less inverter). Such interface systems are used mainly at LV distribution networks. In every case, the assessment of DC components is necessary not only for a proper design of the inverter's control system but also for meeting immunity limits of the interconnection transformer.

The generation of DC components by the inverter under normal operating conditions is mainly due to the faulty operation of the control circuits. One of the basic 
causes of DC injection by inverters is the asymmetry in driving signals of the power electronics. A small amount of DC components is unavoidable as there is always a small asymmetry on the voltages of the three-phase electric grids. Such asymmetries are transferred on the waveforms used for pulse modulation when the filtering process or the extraction of the fundamental voltage component fails. Another cause is measurement errors, especially errors that indicate nonzero values while they are zero. Such errors make the control system generate a small DC current to balance the erroneous non-zero value indicated by the measurement instrument. Obviously, such dc current values are very low (a few mAs) and difficult to be precisely measured, so such errors are very likely when improperly designed measurement instruments are used. Thus, especially under abnormal operating conditions, errors in control system and electronic circuits will allow for a small dc current to flow to the grid before the error is detected and the inverter's operation is shut down [1].

Moreover, DC components may appear in transient conditions in which the inverter's response must be rapid. Such operation is common only in specific inverter types used for the control and mitigation of transient electromagnetic phenomena such as rapid voltage changes (e.g. small and sharp voltage sags, flicker, etc.), voltage waveform distortions (harmonics, transient overvoltages), or rapid power oscillations. Such devices use a special control system of the DC components that may appear on the inverter's output by any cause. Under normal conditions, they use filtering so the reference waveform be purely sinusoidal but no filtering is used at abnormal (transient) conditions where a rapid response is needed [2]. Such a device is the Static Compensator (Statcom), which is used for mitigation of flicker generated by some load types (e.g. arc furnaces) and for mitigation of rapid voltage and power fluctuations on the output of wind parks [3]. It should be noted here that this case does not involve the inverters used for small-scale distributed energy resources as the concept of using such units for the control of fast voltage fluctuations and/or power oscillation damping it is not currently adopted.

The aim of the current paper is the analysis of the way DC components are generated on the output of threephase inverters using the Sinusoidal Pulse Width Modulation (SPWM) technique due to even harmonics. A novel method for the analytical assessment of DC components is presented, which is based on the zero-crossing points of the reference waveform (sinusoidal) and the carrier's waveform (triangular) of SPWM technique. Using realistic values for the SPWM parameters and for the even harmonics, the percentage of DC components of the three line voltages in the inverter's output is calculated. Finally, the values of those parameters, which result in the maximum percentage of DC components, are investigated.

\section{Even Harmonics and DC-Components on Three-Phase Inverter's Output}

2.1. SPWM Technique with Pure Sinusoidal Reference Waveform. Under normal operating conditions of a three-phase



FIGURE 1: Standard three-phase voltage source inverter topology [4].

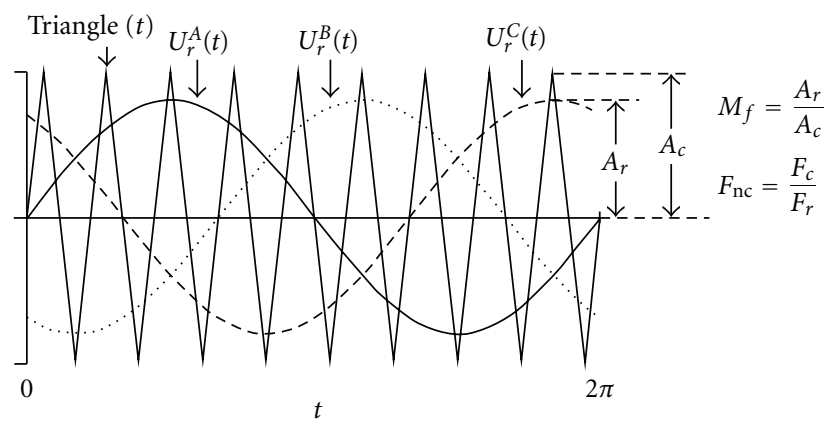

FIGURE 2: Basic waveforms and parameters of SPWM technique.

inverter (Figure 1) that uses SPWM technique, the reference waveform used for the generation of power electronics' firing pulses is formulated based on the fundamental component of the measured network voltage. Thus, any asymmetries or distortions on the network voltage waveform are not transferred on the reference waveform required by the SPWM technique.

In Figure 2, the basic waveforms and parameters of the SPWM technique under normal operating conditions are shown. The reference waveforms are purely sinusoidal and given by the following equations for $A_{c}=1$ :

$$
\begin{gathered}
U_{r}^{A}(t)=M_{f} \cdot \sin (t), \\
U_{r}^{B}(t)=M_{f} \cdot \sin \left(t-\frac{2 \cdot \pi}{3}\right), \\
U_{r}^{C}(t)=M_{f} \cdot \sin \left(t-\frac{4 \cdot \pi}{3}\right),
\end{gathered}
$$

where $U_{r}^{A}(t), U_{r}^{B}(t)$, and $U_{r}^{C}(t)$ are reference waveform functions for phases $A, B$, and $C$; Triangle $(t)$ is the carrier waveform (triangular) function; $A_{r}, F_{r}$ are the amplitude and frequency of reference waveforms; $A_{c}, F_{c}$ are the amplitude and frequency of carrier waveform; $M_{f}=A_{r} / A_{c}$ is the modulation Amplitude Index; $F_{\mathrm{nc}}=F_{c} / F_{r}$ is the modulation Frequency Index.

When a rapid response is required by the inverter, for example, in fast electromagnetic phenomena, the reference waveform is normally formulated in a different way. Specifically, the reference waveform is generated by the measured 


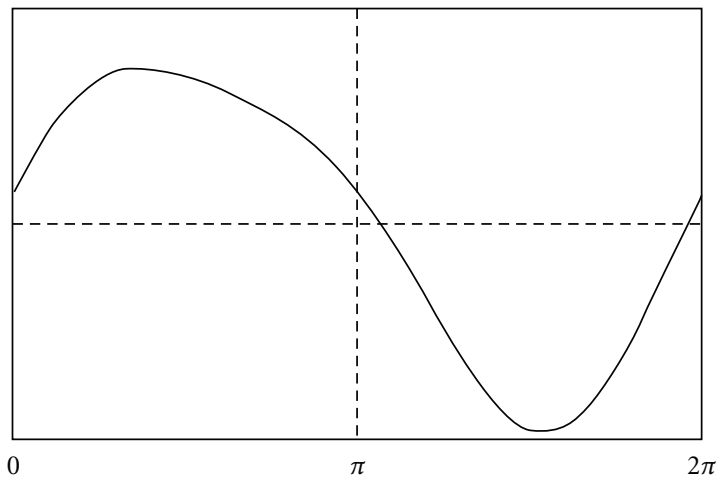

(a)

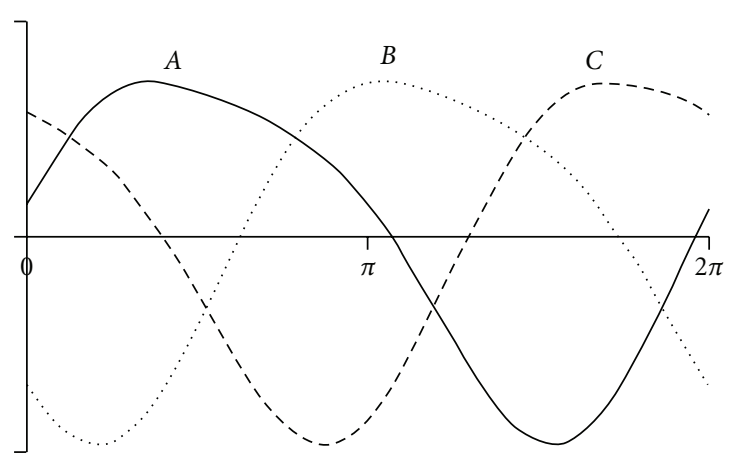

(c)

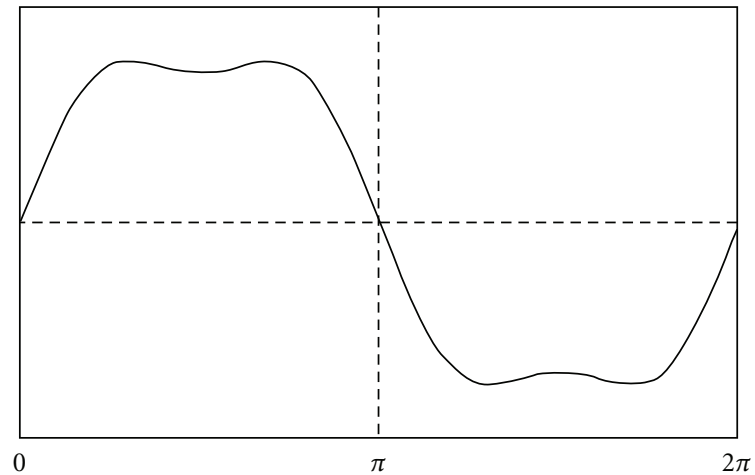

(b)

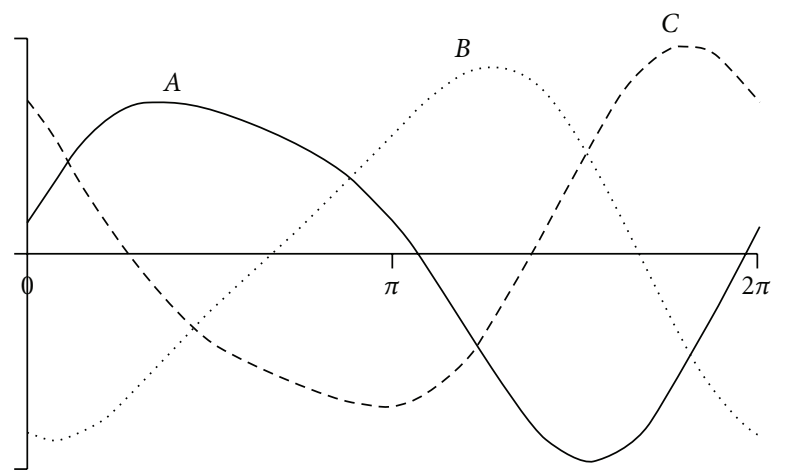

(d)

Figure 3: (a) Waveform with the fundamental and a 2nd harmonic. (b) Waveform with the fundamental and a 3rd harmonic. (c) Waveform with the fundamental and a 2nd harmonic (negative sequence). (d) Waveform with the fundamental and a 2nd harmonic (positive sequence).

network voltage, which is also subjected to 3-phase-to-2phase transformation. The measured network voltage is subjected to filtering only when a rapid response of the inverter is not required, that is, only under normal operating conditions. Therefore, if network voltage contains harmonics and a rapid response of the inverter is needed, no filtering will be applied and the reference waveforms will also contain harmonics [2].

\subsection{SPWM Technique with Asymmetrical Even Harmonics} on the Reference Waveform. A case of asymmetry which can lead to the generation of DC components by an inverter is the presence of even harmonics, especially when there is an asymmetry between the positive and negative half period of the reference waveform [5]. This means that the positive and negative half periods differ at the peak values and duration. This will cause the generation of a DC component on the inverter's output voltage $V^{A O}(t)$ between phase $A$ and the hypothetical point $O$ (Figure 1 ).

In Figures 3(a) and 3(b), the waveforms include a 20\% of a 2nd and 3rd harmonic respectively. The harmonic angle $\phi_{h}$, which is the angle between the harmonic, and the fundamental component, was set at $60^{\circ}$ and $0^{\circ}$, respectively. The asymmetry can be observed only for the 2 nd harmonic not for the 3rd harmonic.
The three reference waveforms of the SPWM technique with the presence of even harmonics without amplitude and angle asymmetry are given by the following equations:

$$
\begin{aligned}
U_{r}^{A}(t)= & M_{f} \cdot \sin (t)+u_{h}^{A}(t) \\
= & M_{f} \cdot \sin (t)+M_{f} \cdot A_{h} \cdot \sin \left[h \cdot\left(t+\frac{\phi_{h}}{h}\right)\right], \\
U_{r}^{B}(t)= & M_{f} \cdot \sin (t)+u_{h}^{B}(t) \\
= & M_{f} \cdot \sin \left(t-\frac{2 \cdot \pi}{3}\right) \\
& +M_{f} \cdot A_{h} \cdot \sin \left[h \cdot\left(t+\frac{\phi_{h}}{h}-\frac{2 \cdot \pi}{3}\right)\right], \\
U_{r}^{C}(t)= & M_{f} \cdot \sin (t)+u_{h}^{C}(t) \\
= & M_{f} \cdot \sin \left(t-\frac{4 \cdot \pi}{3}\right) \\
& +M_{f} \cdot A_{h} \cdot \sin \left[h \cdot\left(t+\frac{\phi_{h}}{h}-\frac{4 \cdot \pi}{3}\right)\right],
\end{aligned}
$$

where $h$ is the harmonic order; $u_{h}^{A}(t), u_{h}^{B}(t)$, and $u_{h}^{C}(t)$ are harmonic functions for phases $A, B$, and $C ; A_{h}$ is the harmonic amplitude as a percentage of the fundamental component; $\phi_{h}$ is the angle between the harmonic and the fundamental component.

Figure 3(c) shows three waveforms with a symmetric 2 nd harmonic. The presence of such a harmonic means that the 

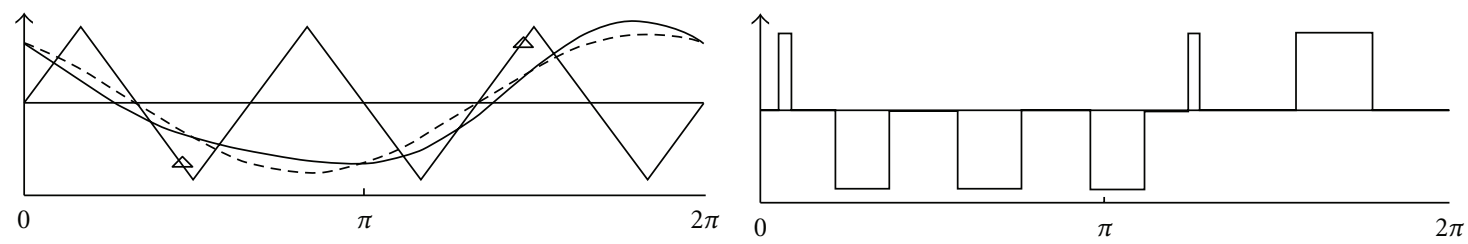

\begin{abstract}
- Waveform with harmonics
- - - Waveform with a 2nd harmonic

$\triangle$ Carrier waveform
\end{abstract}

(a) Reference and carrier waveforms

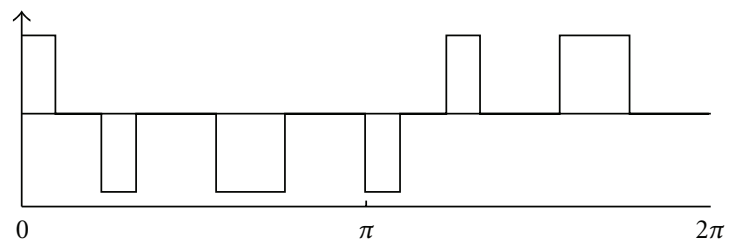

(c) Line voltage for a reference waveform without harmonics (b) Line voltage for a reference waveform with the presence of a 2nd harmonic, DC $=-18.88 \%$

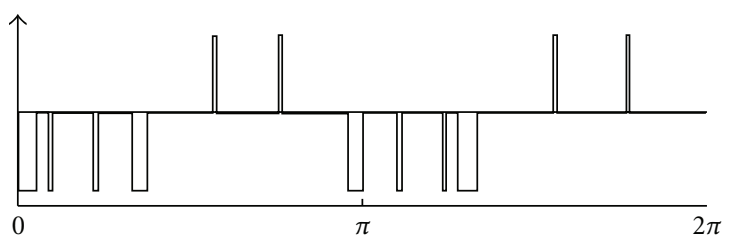

(d) "Difference" (b)-(c)

FIGURE 4: SPWM technique for reference waveform with or without the presence of a 2 nd harmonic. $A_{h}(\%)=20 \%, F_{\mathrm{nc}}=3, \phi_{h}=60^{\circ}$, and $M_{f}=0.9$.

DC component on voltages $V^{A O}(t), V^{B O}(t)$, and $V^{C O}(t)$ will be equal, thus in line voltages $V^{A B}(t), V^{B C}(t)$, and $V^{C A}(t)$ (Figure 1), the DC component will be zero. On the other hand, asymmetrical even harmonics introduce a different asymmetry on the three phases and, consequently, a DC component will appear in line voltages.

The current analysis will consider only the asymmetry in harmonic amplitude. This means that the harmonic amplitude $A_{h}$ of the three waveforms will differ. The bigger the difference is, the higher the asymmetry and the DC component generated by the inverter are. The difference in harmonic amplitude means that the three-phase system contains also a component of inverse (negative) sequence. When a three-phase harmonic component is completely of inverse sequence, the asymmetry is maximized and DC component on the inverter's output is also maximized.

Obviously, the odd or even harmonics are very likely to be asymmetrical in amplitude. This may happen due to DC injection by the inverter for reasons mentioned in the previous section, which will result in generation of even harmonics by the inverter's interconnection transformer [6, 7]. The injected DC current will probably be different in every phase because DC component is different in every line voltage. Thus, the even harmonics generated by the transformer will have different amplitude in every phase, thus they will be asymmetrical.

Figure 3(d) shows a 2nd harmonic of positive (direct) sequence, thus inverse sequence of the normal sequence as 2nd harmonic is by its nature a negative sequence harmonic. This means that the three phases rotate in the $A-C-B$ order and not in the $A-B-C$ order. The 4 th harmonic is normally of positive sequence, the 6 th of zero sequence, and so forth. The same concepts apply also for the odd harmonics. Table 1 lists the normal sequence of even harmonics from the 2 nd to the 10 th order.
TABLE 1: Normal sequence of the first five even harmonics.

\begin{tabular}{ll}
\hline Harmonic order & Sequence of phase components \\
\hline $2 \mathrm{nd}$ & Negative sequence \\
4 th & Positive sequence \\
6 th & Zero sequence \\
8 th & Negative sequence \\
10 th & Positive sequence \\
\hline
\end{tabular}

DC component of output line voltages will be difficult to be observed in the voltage waveform unless it is very high. This could be possible only for asymmetrical even harmonics of inverse sequence and for low Modulation Frequency Index $F_{\text {nc }}$. In Figure 4 , the SPWM technique and one of the line voltages are shown for a purely sinusoidal waveform and for that case it contains a 2nd harmonic. A high harmonic percentage $(20 \%)$ is used for a clear observation of the DC component, and the asymmetries. The Modulation Amplitude Index $M_{f}$ was arbitrarily set to 0.9 and the harmonic angle equals $60^{\circ}$. By observing the line voltages formulated for modulation with a reference waveform containing or not the particular harmonic, we can see a non-zero (Figure 4(b)) and a zero (Figure 4(c)) amount of DC component respectively. This is due to the positive and negative parts which are not identical in pulse series of Figure 4(b) and identical in pulse series of Figure 4(c). This means that the mean value (DC component) will have a nonzero value in the first case and a zero value in the second case.

Another way to verify the existence of a DC component in the line voltage is to obtain the "difference" between the generated voltages formulated when an even harmonic exists or not in the reference waveform by using a simulation package such as the PSCAD/EMTDC [8]. In other words, 


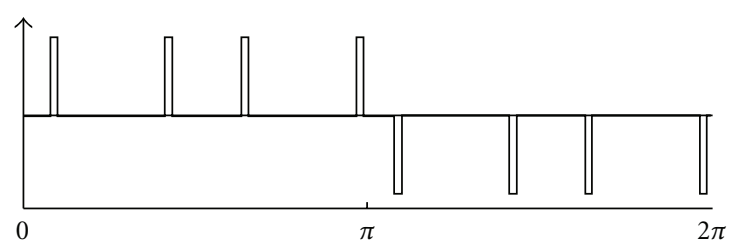

(a) "Difference" for a 3rd harmonic, DC $=0 \%$



(c) "Difference" for an 8th harmonic, DC $=-25.03 \%$

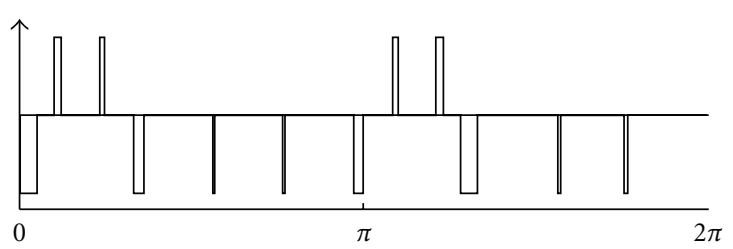

(b) "Difference" for a 6th harmonic, DC $=-10.24 \%$

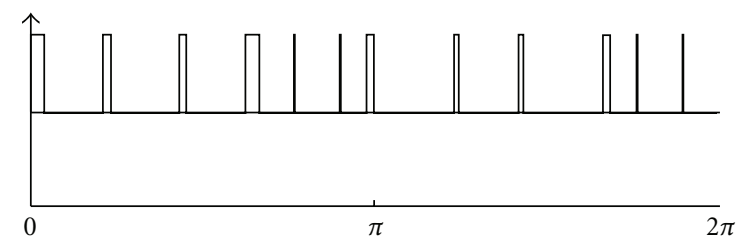

(d) "Difference" for a 10th harmonic, DC $=17.75 \%$

FIGURE 5: "Differences" obtained by the generated line voltages from the modulation with or without the presence of a 3rd, 6th, 8th, and 10th harmonic. $A_{h}(\%)=20 \%, F_{\mathrm{nc}}=3, \phi_{h}=60^{\circ}\left(0^{\circ}\right.$ for the $\left.3 \mathrm{rd}\right)$, and $M_{f}=0.9$.

taking the result by subtracting waveforms of Figures 4(b) and 4(c), the pulse series of Figure 4(d) is formulated. In Figure 5, the "differences" obtained between the generated line voltages from the modulation with or without the presence of a 3rd, 2nd, 6th, 8th, and 10th harmonic are shown, and specifically, the line voltage which gives the maximum DC component. The corresponding DC component value is given as a percentage of the rms value of the fundamental component of the line voltage in the inverter's output, the function of which is mentioned in [4] and given in the following section.

\section{Methodology for the Analytical Calculation of DC Components on Inverter's Output Voltages}

The calculation method of DC components on a three-phase inverter's output voltage is based on the calculation of zerocrossing points of the carrier waveform Triangle $(t)$ for every reference waveform $U_{r}^{A}(t), U_{r}^{B}(t)$, and $U_{r}^{C}(t)$ and can be implemented using a mathematical software package such as MathCad [9]. The zero-crossing points for one period (cycle) are calculated by solving arithmetically the following equation:

$$
U_{r}(t)-\text { Triangle }(t)=0 .
$$

In order to solve this equation, an analytical expression is required for the triangular waveform Triangle $(t)$. It can be easily observed that the triangular waveform consists of $n$ direct lines, where $n=2 \cdot F_{\mathrm{nc}}$. The equation that describes the triangular waveform Triangle $(t)$ for one cycle $(t \in[0,2 \pi])$ can be written for $A_{c}=1$ as follows:

$$
\begin{aligned}
& \text { Triangle }(t)=(-1)^{n+1} \\
&+(-1)^{n} \cdot\left[t-\left(n-\frac{1}{2}\right) \cdot \frac{\pi}{F_{\mathrm{nc}}}\right] \cdot \frac{2 \cdot F_{\mathrm{nc}}}{\pi}, \\
& n=0,1, \ldots, 2 \cdot F_{\mathrm{nc}}, \quad t \in\left[\frac{(2 n-1) \cdot \pi}{2 \cdot F_{\mathrm{nc}}}, \frac{(2 n+1) \cdot \pi}{2 \cdot F_{\mathrm{nc}}}\right] .
\end{aligned}
$$

The zero-crossing points of the triangular waveform with the reference waveforms define voltages $V^{A O}(t), V^{B O}(t)$, and $V^{C O}(t)$ (Figure 1) according to the following relations:

$$
V(t)=\left\{\begin{aligned}
\frac{U_{d}}{2}: & U_{r} \geq \operatorname{Triangle}(t) \\
& \Longrightarrow t \in\left[t_{2 n}, t_{2 n-1}\right], \quad n=1,2, \ldots, F_{\mathrm{nc}}, \\
-\frac{U_{d}}{2}: U_{r}<\operatorname{Triangle}(t) & \\
& \Longrightarrow t \in\left[t_{2 n+1}, t_{2 n}\right], \quad n=0,1, \ldots, F_{\mathrm{nc}}-1,
\end{aligned}\right.
$$

where $U_{d}$ is the voltage on the dc side of the inverter (Figure 1) $t_{0}, t_{1}, \ldots, t_{2} \cdot F_{\mathrm{nc}}$ are the time points corresponding to zero-crossing points of the triangular waveform with the reference waveforms.

Using the time points given by (5), the inverter's voltage pulse series $V^{A O}(t), V^{B O}(t)$, and $V^{C O}(t)$ can be formulated. From them, the DC component can be calculated by taking the mean time for a period. Specifically, the pulse duration for the positive and negative part of the pulse series is added and multiplied with their amplitude $U_{d} / 2$. The resulting summations are subtracted and the result is divided with the period $2 \pi$. The voltage value $U_{d}$ does not affect the value of the DC component, so it can be set equal to 1 .

The analytical equations for the DC component ratio of inverter's voltages $V^{A O}(t), V^{B O}(t)$, and $V^{C O}(t)$ with the rms fundamental line voltage at the inverter's output $V_{o, 1(\mathrm{rms})} \mathrm{can}$ be written as follows:

$$
\begin{aligned}
& V_{\mathrm{dc}}^{A O} \\
& =\frac{(1 / 2 \pi)\left[-\sum_{n=0}^{F_{\mathrm{nc}}-1}\left(t_{2 n+1}^{A}-t_{2 n}^{A}\right) / 2+\sum_{n=1}^{F_{\mathrm{nc}}}\left(t_{2 n}^{A}-t_{2 n-1}^{A}\right) / 2\right]}{V_{o, 1(\mathrm{rms})}}, \\
& V_{\mathrm{dc}}^{B O} \\
& =\frac{(1 / 2 \pi)\left[-\sum_{n=0}^{F_{\mathrm{nc}}-1}\left(t_{2 n+1}^{B}-t_{2 n}^{B}\right) / 2+\sum_{n=1}^{F_{\mathrm{nc}}}\left(t_{2 n}^{B}-t_{2 n-1}^{B}\right) / 2\right]}{V_{o, 1(\mathrm{rms})}},
\end{aligned}
$$




$$
\begin{aligned}
& V_{\mathrm{dc}}^{C O} \\
& =\frac{(1 / 2 \pi)\left[-\sum_{n=0}^{F_{\mathrm{nc}}-1}\left(t_{2 n+1}^{C}-t_{2 n}^{C}\right) / 2+\sum_{n=1}^{F_{\mathrm{nc}}}\left(t_{2 n}^{C}-t_{2 n-1}^{C}\right) / 2\right]}{V_{o, 1(\mathrm{rms})}} .
\end{aligned}
$$

The rms value of the fundamental component of the inverter's output line voltage is given as follows $[4,10]$ :

$$
V_{o, 1(\mathrm{rms})}=\frac{\sqrt{3}}{2 \sqrt{2}} \cdot M_{f} \cdot U_{d}
$$

For the line voltages $V^{A B}(t), V^{B C}(t)$ and $V^{C A}(t)$, the DC component is calculated as follows:

$$
\begin{aligned}
& V_{\mathrm{dc}}^{A B}=V_{\mathrm{dc}}^{A O}-V_{\mathrm{dc}}^{B O}, \\
& V_{\mathrm{dc}}^{B C}=V_{\mathrm{dc}}^{B O}-V_{\mathrm{dc}}^{C O}, \\
& V_{\mathrm{dc}}^{C A}=V_{\mathrm{dc}}^{C O}-V_{\mathrm{dc}}^{A O} .
\end{aligned}
$$

The SPWM technique with an inverse (positive) sequence 8 th harmonic on the reference waveforms when $A_{h}(\%)=20 \%, F_{\mathrm{nc}}=9, \phi_{h}=60^{\circ}$, and $M_{f}=0.8$ is shown in Figure 6. The zero-crossing (time) points of the reference waveforms and the triangular waveform have been calculated using MathCad software [9] and are given in Table 2. The values of the DC component for the voltages $V^{A O}(t), V^{B O}(t)$, and $V^{C O}(t)$ as a percentage of the rms value of the fundamental component of the inverter's output line voltage $V_{o, 1(\mathrm{rms})}$ are approximately as follows:

$$
V_{\mathrm{dc}}^{A O} \cong 7.177 \%, \quad V_{\mathrm{dc}}^{B O} \cong 0 \%, \quad V_{\mathrm{dc}}^{C O} \cong-7.177 \% .
$$

The corresponding values for the line voltages $V^{A B}(t)$, $V^{B C}(t)$, and $V^{C A}(t)$ are as follows:

$$
\begin{gathered}
V_{\mathrm{dc}}^{A B}=V_{\mathrm{dc}}^{A O}-V_{\mathrm{dc}}^{B O} \cong 7.177 \%, \\
V_{\mathrm{dc}}^{B C}=V_{\mathrm{dc}}^{B O}-V_{\mathrm{dc}}^{C O} \cong 7.177 \%, \\
V_{\mathrm{dc}}^{C A}=V_{\mathrm{dc}}^{C O}-V_{\mathrm{dc}}^{A O} \cong-14.354 \% .
\end{gathered}
$$

\section{Assessment of DC Components}

The DC component for every line voltage at the inverter's output $V_{\mathrm{dc}}^{A B}, V_{\mathrm{dc}}^{B C}$, and $V_{\mathrm{dc}}^{C A}$ will probably have different value. It may take positive or negative values as we saw in the previous section. As the sign is not of significance, the calculation of DC component will be based on the maximum absolute value among the three DC values for

\begin{tabular}{|c|c|c|c|}
\hline$U_{r}^{A}(t)$ & $\wedge$ Triangle $(t)$ & $U_{r}^{B}(t) \wedge$ Triangle $(t)$ & $U_{r}^{C}(t) \wedge$ Triangle $(t)$ \\
\hline$t_{0} \cong$ & 0.031 & -0.134 & 0.095 \\
\hline$t_{1} \cong$ & 0.318 & 0.479 & 0.274 \\
\hline$t_{2} \cong$ & 0.829 & 0.552 & 0.748 \\
\hline$t_{3} \cong$ & 0.912 & 1.134 & 1.072 \\
\hline$t_{4} \cong$ & 1.559 & 1.299 & 1.379 \\
\hline$t_{5} \cong$ & 1.581 & 1.774 & 1.867 \\
\hline$t_{6} \cong$ & 2.202 & 2.094 & 1.987 \\
\hline$t_{7} \cong$ & 2.322 & 2.415 & 2.608 \\
\hline$t_{8} \cong$ & 2.810 & 2.890 & 2.630 \\
\hline$t_{9} \cong$ & 3.117 & 3.055 & 3.277 \\
\hline$t_{10} \cong$ & 3.441 & 3.637 & 3.360 \\
\hline$t_{11} \cong$ & 3.915 & 3.710 & 3.871 \\
\hline$t_{12} \cong$ & 4.094 & 4.323 & 4.158 \\
\hline$t_{13} \cong$ & 4.661 & 4.434 & 4.473 \\
\hline$t_{14} \cong$ & 4.775 & 4.947 & 4.958 \\
\hline$t_{15} \cong$ & 5.351 & 5.236 & 5.120 \\
\hline$t_{16} \cong$ & 5.514 & 5.525 & 5.696 \\
\hline$t_{17} \cong$ & 5.999 & 6.038 & 5.811 \\
\hline$t_{18} \cong$ & 6.314 & 6.149 & 6.378 \\
\hline
\end{tabular}
every line voltage or the voltage between each phase and the hypothetical point $O$ (Figure 1), that is,

$$
\begin{aligned}
& \max \left\{\left|V_{\mathrm{dc}}^{A O}\right|,\left|V_{\mathrm{dc}}^{B O}\right|,\left|V_{\mathrm{dc}}^{C O}\right|\right\} \text { or } \\
& \max \left\{\left|V_{\mathrm{dc}}^{A B}\right|,\left|V_{\mathrm{dc}}^{B C}\right|,\left|V_{\mathrm{dc}}^{C A}\right|\right\} .
\end{aligned}
$$

The value of the DC component depends on parameters of the SPWM technique and the even harmonics. The assessment of the maximum DC percentage will be done for
TABle 2: Time instants based on zero-crossings points of reference waveforms and triangular waveform.

a wide range of possible values of those parameters and the effect of every parameter will be investigated.

4.1. Parameters of the SPWM Technique. The basic parameters and the corresponding range that will be used in the current analysis are as follows.

Modulation Frequency Index $F_{n c}$. This parameter is an odd multiple of 3 for three-phase inverters $[4,10]$ in order to eliminate the undesirable odd harmonics. Its value depends on the nominal power of the inverter. Inverters of high power use a low value of $F_{\mathrm{nc}}$, mainly for power losses elimination reasons and to increase the efficiency. The current analysis will include every possible inverter size that may be connected to MV or LV level. Thus, the $F_{\mathrm{nc}}$ will take values in the range $9-159$ or in the range $450-7950 \mathrm{~Hz}$ (corresponding frequency values).

Modulation Amplitude Index $M_{f}$. The range is theoretically between 0 and 1 but common values are in the range 0.6-1.

4.2. Parameters of the Even Harmonics. The basic parameters and the corresponding range that will be used in the current analysis are as follows.

Harmonic Order $h$. The even harmonics that are more likely to appear are the low order harmonics between the 2nd and the 10 th harmonic. The 2 nd harmonic gathers the most 

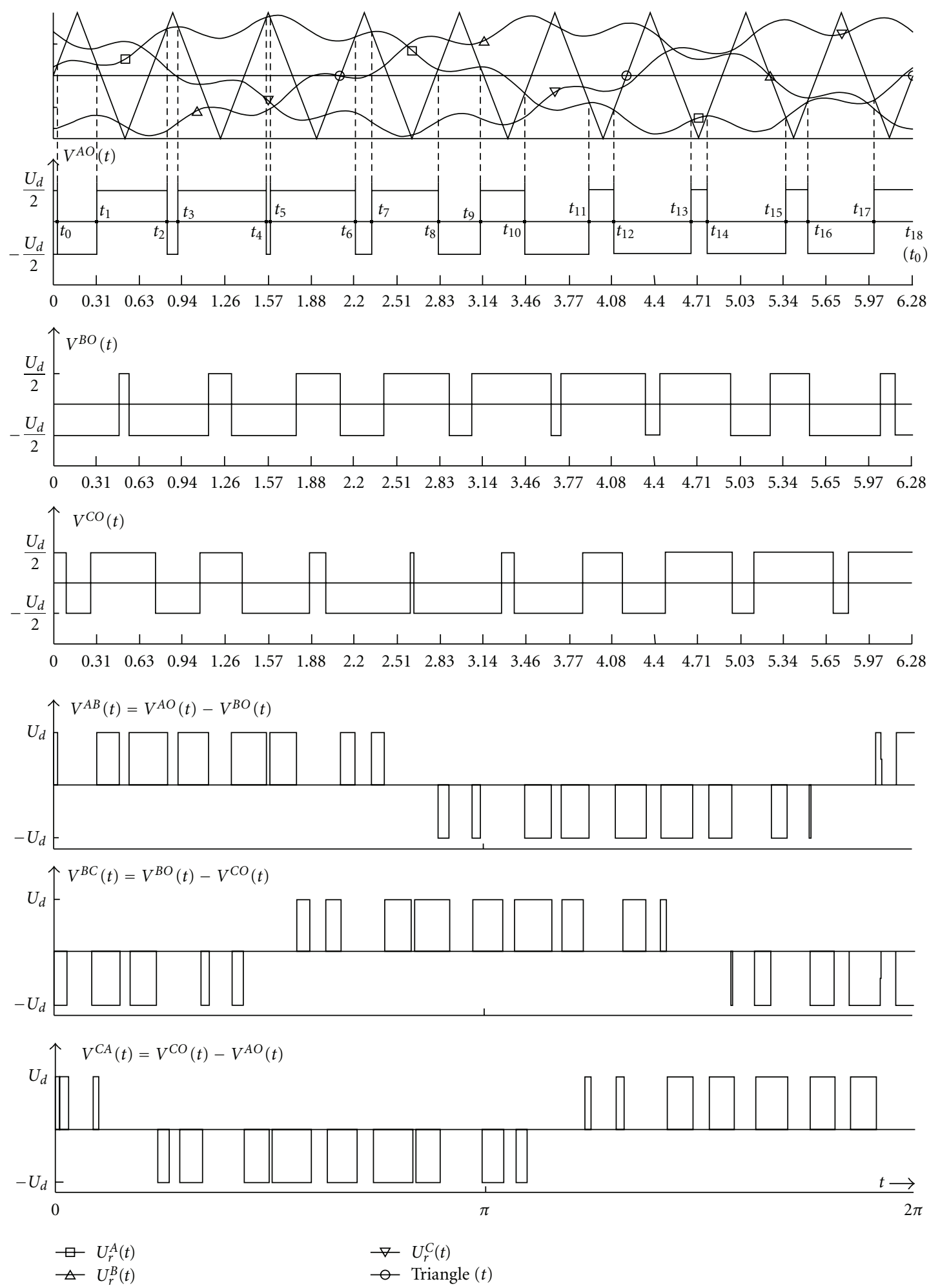

FIGURE 6: Reference waveforms with an 8th harmonic and zero crossings with the triangular waveform obtained using PSCAD/EMTDC simulation package [8]. $A_{h}(\%)=20 \%, F_{\mathrm{nc}}=9, \phi_{h}=60^{\circ}$, and $M_{f}=0.8$. 
TABLE 3: Approximate relation for the maximum DC component versus the harmonic percentage.

\begin{tabular}{|c|c|c|c|}
\hline Harmonic order & $F_{\text {nc }}$ & $\begin{array}{l}\text { Maximum DC component on voltages } \\
\qquad V^{A O}(t), V^{B O}(t) \text {, and } V^{C O}(t)\end{array}$ & $\begin{array}{l}\text { Maximum DC component on voltages } \\
\qquad V^{A B}(t), V^{B C}(t) \text {, and } V^{C A}(t)\end{array}$ \\
\hline 2nd & $9-159$ & $\cong 0.01 \cdot A_{h}$ & $\cong 0.035 \cdot A_{h}$ \\
\hline 4 th & $9-159$ & $\cong 0.008 \cdot A_{h}$ & $\cong 0.03 \cdot A_{h}$ \\
\hline 6th & $9-159$ & $\cong 0.007 \cdot A_{h}$ & $\cong 0.03 \cdot A_{h}$ \\
\hline 8th & $15-159$ & $\cong 0.006 \cdot A_{h}$ & $\cong 0.02 \cdot A_{h}$ \\
\hline 10th & $15-159$ & $\cong 0.005 \cdot A_{h}$ & $\cong 0.02 \cdot A_{h}$ \\
\hline 8 th or 10 th & 9 & $\cong 0.09 \cdot A_{h}$ & $\cong 0.42 \cdot A_{h}$ \\
\hline
\end{tabular}



Figure 7: Reference waveforms with the fundamental and an asymmetric harmonic $\left(\phi_{h}=90^{\circ}, M_{f}=0.8\right)$.

references in the literature $[5-7,11-13]$ as it is the most frequent and usually takes higher values than the other even harmonics.

Harmonic Amplitude $A_{h}$. The amplitude of even harmonics on network voltage is usually low under normal operating conditions. Thus, possible values according to some references $[2,5]$ are between $1 \%$ and $3 \%$ of the network voltage fundamental component amplitude, and the 2nd harmonic is more likely to keep those levels. The other examined even harmonics (4th to 10th) rarely present those levels except for abnormal operation of power system elements or transient conditions. Specifically, in cases of energization of high power transformers [2, 12] or injection of large amounts of DC current (e.g., by geomagnetically induced currents) $[6,11,13]$, large amounts of even harmonics are generated but for a short time period.

Harmonic Angle $\phi_{h}$. The angle between the harmonic and the fundamental component will take values in the range $0^{\circ}-$ $180^{\circ}$.

Asymmetry of the Harmonics. The asymmetry assumed in the current analysis considers the amplitude difference of harmonics in every network phase voltage. Possible values for this difference can be assumed to be about $20 \%$ at maximum. Such an asymmetry may appear on the even harmonics generated by a power transformer when affected by a dc current injected by the inverter. This current will be different in every phase $A, B$, and $C$ as it happens with the DC components of the line voltages. Consequently, the even harmonics generated by the power transformer will have different amplitude in every phase, thus they will be asymmetrical.

All the above parameters and the value range used in the current analysis are summarized in Table 3. The analytical equations for the reference waveforms $U_{r}^{A}(t), U_{r}^{B}(t)$, and $U_{r}^{C}(t)$ when they contain an even harmonic with $20 \%$ lower amplitude for phase $B$ in relation with phases $A$ and $C$, are as follows:

$$
\begin{aligned}
& U_{r}^{A}(t)= M_{f} \cdot \sin (t)+M_{f} \cdot A_{h} \cdot \sin \left[h \cdot\left(t+\frac{\phi_{h}}{h}\right)\right], \\
& \begin{aligned}
U_{r}^{B}(t)= & M_{f} \cdot \sin \left(t-\frac{2 \cdot \pi}{3}\right) \\
& +0.8 \cdot M_{f} \cdot A_{h} \cdot \sin \left[h \cdot\left(t+\frac{\phi_{h}}{h}-\frac{2 \cdot \pi}{3}\right)\right], \\
U_{r}^{C}(t)= & M_{f} \cdot \sin \left(t-\frac{4 \cdot \pi}{3}\right) \\
& +M_{f} \cdot A_{h} \cdot \sin \left[h \cdot\left(t+\frac{\phi_{h}}{h}-\frac{4 \cdot \pi}{3}\right)\right] .
\end{aligned}
\end{aligned}
$$

The reference waveforms with a 2 nd harmonic and such an asymmetry are shown in Figure 7 . The difference on the maximum (peak) value between phases $A$ (or $C$ ) and $B$ in the positive and negative part of the waveform can be easily observed.

Applying the methodology of the previous section, the DC component of the inverter's line voltages is calculated. By carefully examining (12) and taking into account those mentioned in the previous Sections, it can be easily proved that the DC component will be equal in absolute terms for line voltages $V^{A B}$ and $V^{B C}$ and zero for the line voltage $V^{C A}$. Thus, only one of the components $\left|V_{\mathrm{dc}}^{A B}\right|$ and $\left|V_{\mathrm{dc}}^{B C}\right|$ has to be calculated.

\section{Results and Discussion}

The results for the maximum DC component are given as a percentage of the rms value of the inverter's line voltage fundamental component $V_{o, 1(\mathrm{rms})}$. The DC component was calculated using MathCad [9] not only for line voltages but also for the voltages between every phase and point $O$ (Figure 1). Among the three calculated values, the maximum was taken. It should be noted here that the DC component 



(a)
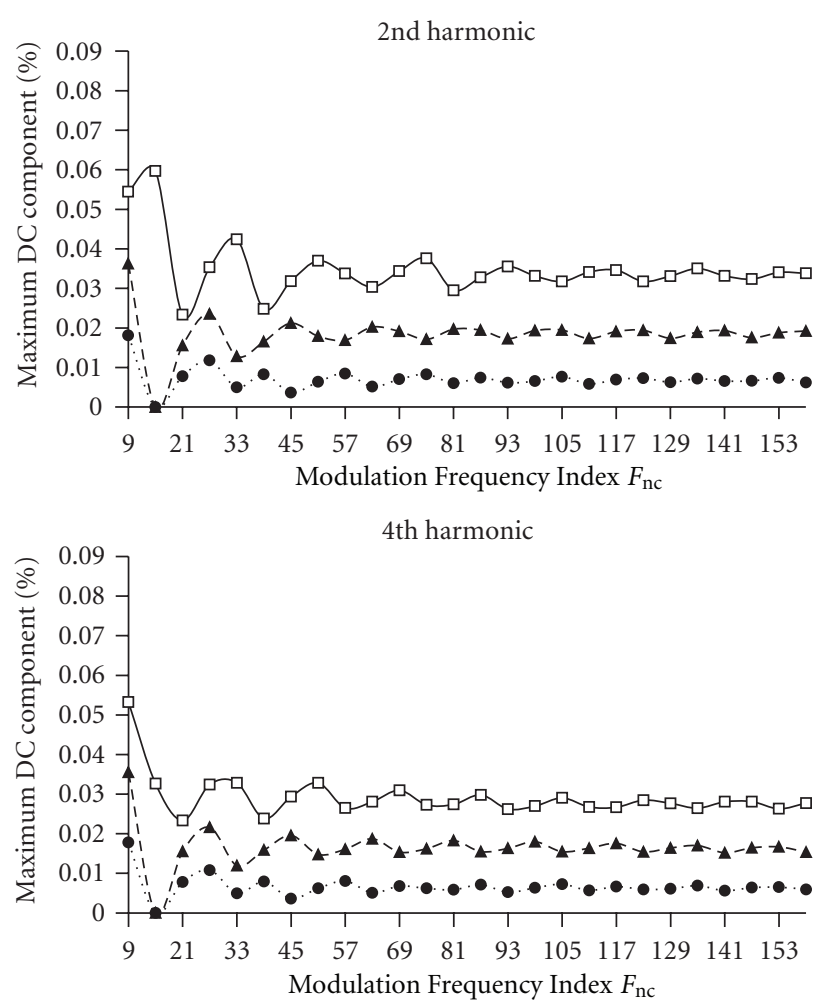

6th harmonic



(b)

Figure 8: (a) Maximum DC component on voltages $V^{A O}(t), V^{B O}(t)$, and $V^{C O}(t)$ for a 2nd, 4th, or 6th harmonic and $F_{\mathrm{nc}}=9-159$. (b) Maximum DC component on voltages $V^{A B}(t), V^{B C}(t)$, and $V^{C A}(t)$ for a 2 nd, 4 th, or 6 th harmonic and $F_{\mathrm{nc}}=9-159$.

of the voltages between each phase and point $O$ increases as the harmonic percentage increases while the DC component on the line voltages increases as the asymmetry of harmonics increases. If no asymmetry exists, the DC component on the line voltages becomes zero independently of the harmonic percentage.

In Figure 8, the maximum DC component is shown for every possible combination of the parameters discussed in the previous Section when a 2nd, 4th, or 6th harmonic is present on the SPWM reference waveforms. It can be seen that the DC component is higher for the voltages between each phase and point $O$ (Figure 8 ) in relation with the line voltages (Figure 9), which was expected to occur. It can also be observed that the 2nd harmonic gives the higher DC component among the others followed by the 4th harmonic. As it was expected, the DC component increases as the harmonic percentage increases. For $F_{\mathrm{nc}}=15$ and low harmonic percentage ( $1 \%$ or $2 \%$ ), the DC component is almost zero and takes non-zero value only for a $3 \%$ harmonic percentage. Moreover, the DC component takes almost equal values for large values of $F_{\text {nc }}$.

Figure 9 shows the maximum DC component when an 8 th or 10th harmonic is present on the reference waveforms. Only the values for $F_{\mathrm{nc}}$ between 15 and 159 are given in this 



(a)

(b)

Figure 9: (a) Maximum DC component on voltages $V^{A O}(t), V^{B O}(t)$, and $V^{C O}(t)$ for an 8th or 10th harmonic and $F_{\mathrm{nc}}=15-159$. (b) Maximum DC component on voltages $V^{A B}(t), V^{B C}(t)$, and $V^{C A}(t)$ for an 8 th or 10 th harmonic and $F_{\mathrm{nc}}=15-159$.

figure as the DC component follows a different pattern for $F_{\text {nc }}=9$. The DC component increases similarly with the low order harmonics (2nd-6th) decreases as the harmonic order increases. Moreover, the maximum DC component increases as the harmonic percentage increases. Similarly with the lower order harmonics, for a low harmonic percentage $(1 \%$ or $2 \%$ ), the DC component is almost zero. Furthermore, as for low order harmonics, the DC component takes almost equal values for large values of $F_{\text {nc }}$.

Figure 10 depicts the maximum DC component when a 8 th or 10th harmonic is present on the reference waveforms for $F_{\mathrm{nc}}=9$. In this case, the DC component takes much higher values than for the other harmonics and the other values of $\mathrm{F}_{\mathrm{nc}}$ and is the same for these two harmonics (8th and 10th). It can be concluded that these two harmonics dominate as regards the DC component and they can be expressed by the relationship $F_{\mathrm{nc}} \pm 1$.

By close examination of the results given in Figures 8-10, an approximate relation can be written for the maximum DC component versus the harmonic percentage on the reference waveforms of SPWM technique, which is presented in Table 3.

Moreover, the value of Modulation Amplitude Index $M_{f}$, at which the maximum DC component occurs, follows the same pattern for all the examined harmonics except for the case of 8th and 10th harmonic and for $F_{\mathrm{nc}}=9$. Specifically, the maximum (for all the examined parameters) DC component increases as the $M_{f}$ increases and becomes maximum for $M_{f}=1$. In particular, in most cases the DC component is zero for $M_{f}<0.9$. A typical variation pattern for the DC component versus $M_{f}$ is shown in Figure 11.

The respective variation for the 8 th and 10th harmonic and for $F_{\mathrm{nc}}=9$ is shown in Figure 12. Unlike the pattern shown in Figure 11, in this case the value of DC component ascends until a maximum value at about $M_{f}=0.98$ and descends until $M_{f}=1$.

Finally, Figure 13 depicts the typical pattern followed by the DC component in relation with the harmonic angle $\phi_{h}$ in the majority of cases. For $\phi_{h}=90^{\circ}$, the DC component becomes maximum while the values $0^{\circ}$ and $180^{\circ}$ give the same percentage of DC component in absolute terms.

\section{Conclusions}

In this paper, the amount of DC components on the output voltages of inverter-based renewable energy resources was investigated when one of the first five even harmonics 


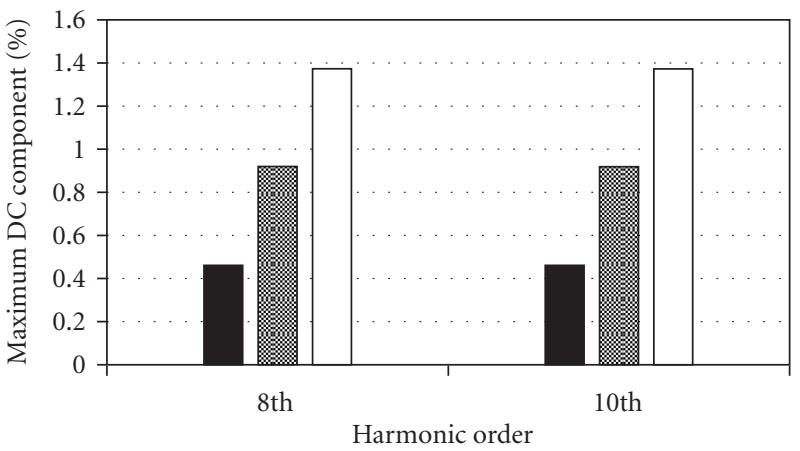

$A_{h}=1 \%$

娄 $A_{h}=2 \%$

$\square A_{h}=3 \%$

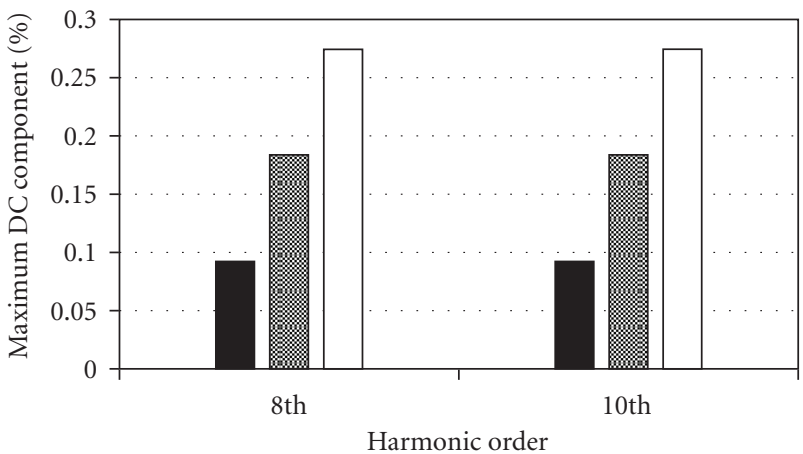

$A_{h}=1 \%$

圈 $A_{h}=2 \%$

$\square A_{h}=3 \%$

(a)

(b)

Figure 10: (a) Maximum DC component on voltages $V^{A O}(t), V^{B O}(t)$, and $V^{C O}(t)$ for an 8th or 10th harmonic and $F_{\text {nc }}=9$. (b) Maximum DC component on voltages $V^{A B}(t), V^{B C}(t)$, and $V^{C A}(t)$ for an 8 th or 10 th harmonic and $F_{\mathrm{nc}}=9$.

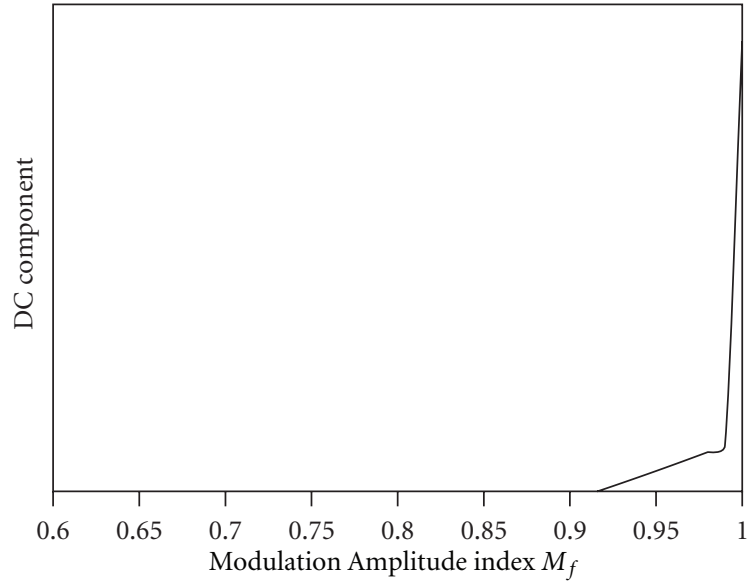

FIgUre 11: Typical variation of the DC component in relation with the Modulation Amplitude Index $M_{f}$ for the 2nd, 4th, and 8th harmonics when $F_{\mathrm{nc}}=9-159$ but also for the 8th and 10th harmonics when $F_{\mathrm{nc}}=15-159$.

appears on the network voltage. A calculation method of the DC components was developed based on the zero crossings of the SPWM control waveforms. The results for the DC component were given as a percentage of the rms fundamental component of the inverter's output voltage. The absolute maximum value among the three per phase or line voltages was taken in order to investigate the maximum levels of DC component. All the parameters of SPWM technique and even harmonics were examined and the maximum range of values was used.

It can be concluded by the current analysis that the DC components on the inverter's output voltage are not considerable under normal operating conditions and do not constitute a problem even for high penetration of distributed energy resources to the electric grid. This is supported by the fact that the values of the DC components assessed here are the maximum expected and only when a

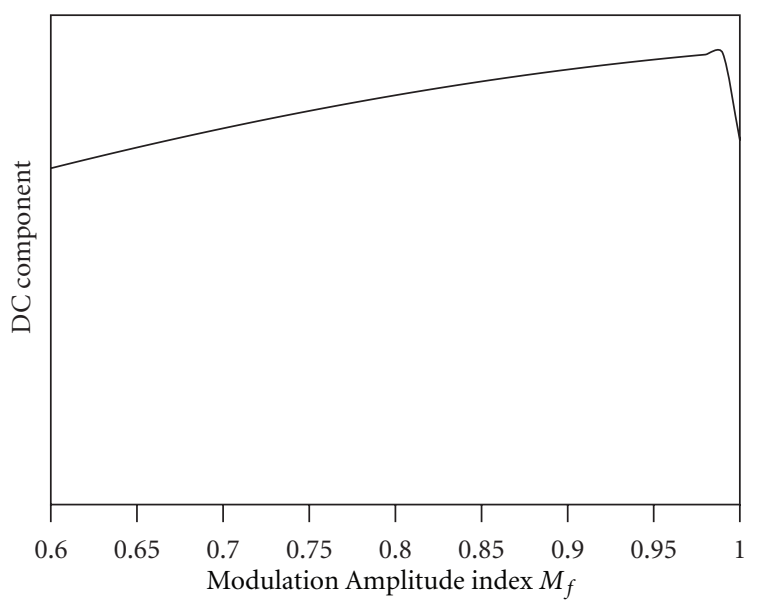

FIGURE 12: Typical variation of the DC component in relation with the Modulation amplitude index $M_{f}$ for the 8th and 10th harmonics when $F_{\mathrm{nc}}=9$.

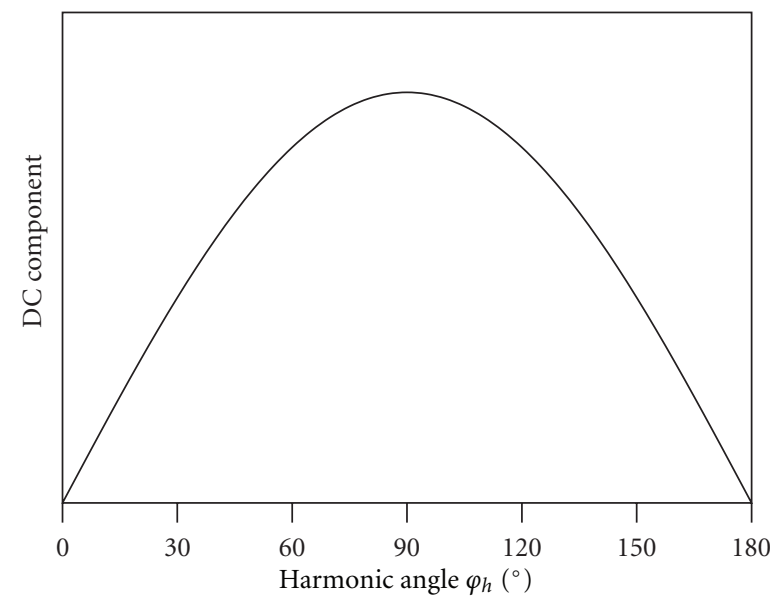

FIGURE 13: Typical variation of the DC component in relation with the harmonic angle $\phi_{h}$ for all even harmonics. 
series of coincidences occurs, a considerable amount of DC components may appear. Moreover, as more sophisticated topologies and control systems are used for the inverterbased interface system of the renewable energy resources, the DC injection will be minor. The only exception found by the current analysis which gives a considerable amount of DC components is the case for $F_{\mathrm{nc}}=9$ together with the presence of an 8 th or 10 th harmonic.

\section{Acknowledgments}

This paper was prepared during a project funded by the European Union and Greek Government. The authors wish to acknowledge the significant contribution of the sponsors.

\section{References}

[1] E. Spooner, "A New Australian Standard for Small Gridconnected Renewable Generation Systems Connected via Inverters," 2001, http://www.acre.ee.unsw.edu.au/acrelab/ Assets/Papers/142_Spooner_AUPEC01_Final2.pdf.

[2] T. Nakajima, K. I. Suzuki, M. Yajima, N. Kawakami, K. I. Tanomura, and S. Irokawa, "A new control method preventing transformer DC magnetization for voltage source selfcommutated converters," IEEE Transactions on Power Delivery, vol. 11, no. 3, pp. 1522-1528, 1996.

[3] K. H. Sobrink, N. Jenkins, F. C. A. Schettler, J. Pedersen, K. O. H. Pedersen, and K. Bergmann, "Reactive power compensation of a $24 \mathrm{MW}$ wind farm using a 12-pulse voltage source converter," in Proceedings of the International Conference Large High Voltage Electric Systems (CIGRÉ '98), 1998.

[4] M. Rashid, Power Electronics Handbook, 3rd edition, 2011.

[5] J. A. Orr and A. E. Emanuel, "On the need for strict second harmonic limits," IEEE Transactions on Power Delivery, vol. 15, no. 3, pp. 967-971, 2000.

[6] S. Lu, Y. Liu, and J. D. L. Ree, "Harmonics generated from a DC biased transformer," IEEE Transactions on Power Delivery, vol. 8, no. 2, pp. 725-731, 1993.

[7] E. V. Larsen, R. A. Walling, and C. J. Bridenbaugh, "Parallel AC/DC transmission lines steady-state induction issues," IEEE Transactions on Power Delivery, vol. 4, no. 1, pp. 667-674, 1989.

[8] PSCAD/EMTDC User's Guide, "Manitoba HVDC Research Centre," Version 4. 7, 2010.

[9] MathCad User's Guide, "Parametric Technology Corporation (PTC)," Version 14, 2007.

[10] N. Mohan, T. Undeland, and W. Robbins, Power Electronics: Converters, Applications, and Design, John Wiley \& Sons, New York, NY, USA, 1995.

[11] P. R. Price, "Geomagnetically induced current effects on transformers," IEEE Transactions on Power Delivery, vol. 17, no. 4, pp. 1002-1008, 2002.

[12] E. Styvaktakis, Automating Power Quality Analysis [Ph.D. thesis], Chalmers University of Technology, Gothenburg, Sweden, 2002.

[13] W. Xu, T. G. Martinich, J. H. Sawada, and Y. Mansour, "Harmonics from SVC transformer saturation with direct current offset," IEEE Transactions on Power Delivery, vol. 9, no. 3, pp. 1502-1509, 1994. 

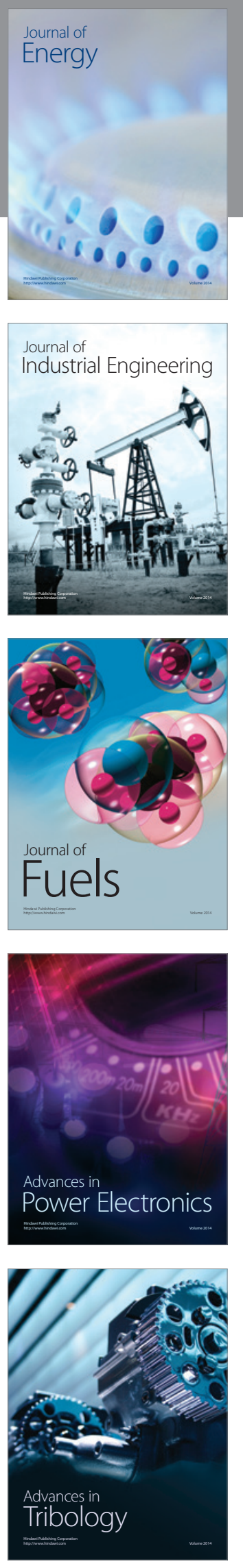
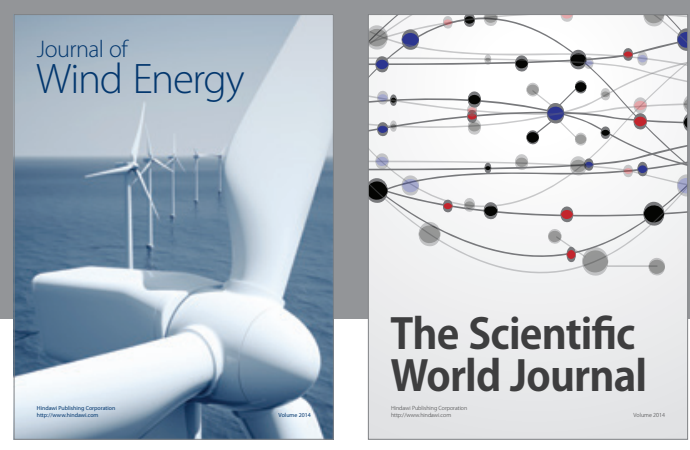

The Scientific World Journal

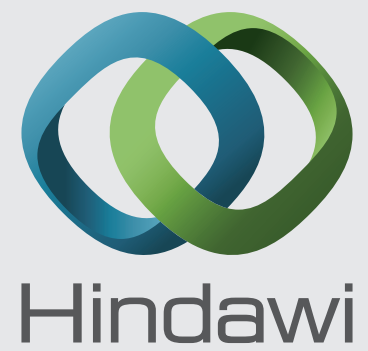

Submit your manuscripts at http://www.hindawi.com


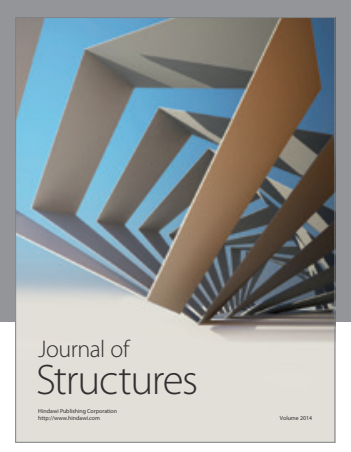

Rotating
Mechinery
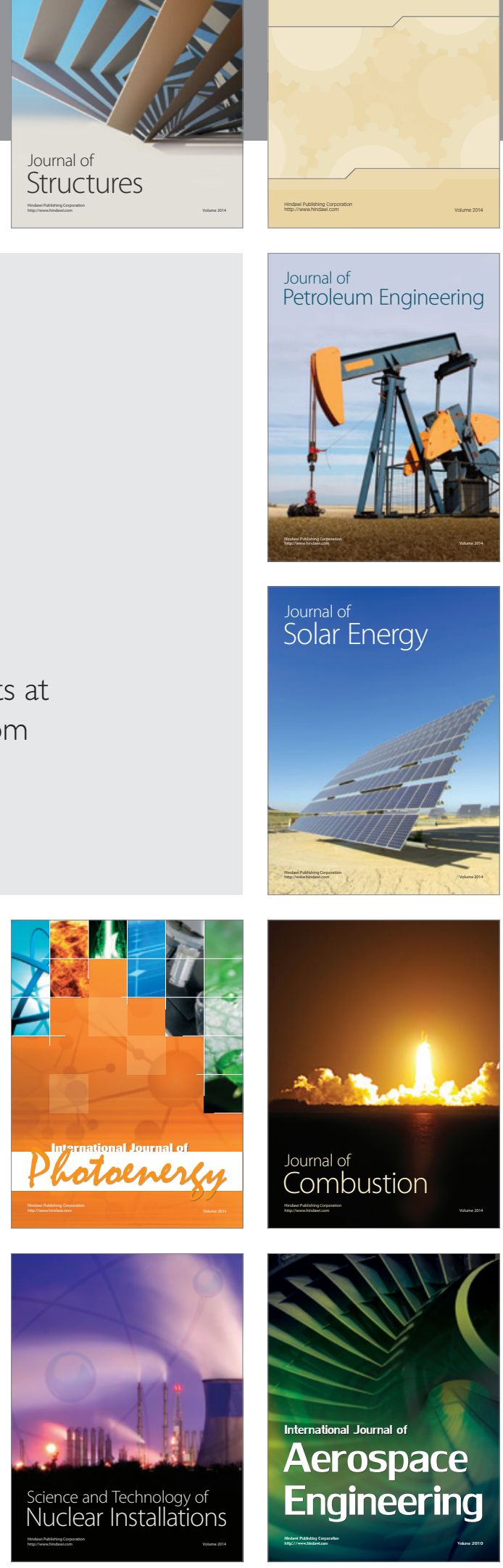